\title{
Problembezeichnung und Problemerlebnis - Gedanken zum problematischen Selbstverständnis einer Übersetzungswissenschaft
}

\author{
Ismail Işcen (Mersin)
}

\begin{abstract}
This study, related to the discussion concerning the basis of an "ÜW" (Science of Translation), aims to show the connection between the problems, which are concretely lived in the praxis of studies and researches (concentrated on the general topic "translation"), and the definitions of these problems. The main arguments, which have been expressed in particular since the early 80's, can be described as against or for the "pure" disciplinary structure of a science of translation (ÜW). The study tries to make clear that these arguments have their origin in other disciplines, which aim to incorporate the translation researches.

Concerning the theoretical basis of an ÜW (as an opposite/alternative to TS, translation studies) the study discusses the possibilities given by the arguments named, particularly those emphasizing the precipice between the "Ü-Theorie" and "Ü-Praxis", the interdisciplinary spectrum of several sciences, the complexity of the subject etc.

At the end of the study a proposal is formulated for resolving the main problem (of the discussion itself) based on extra-disciplinary approaches. The discussion on the basis of a science of Translation (ÜW) must be clarified by a logical order of proceedings and the "dynamic structure" of principle analyses in ÜW, which can be defined as subordination and coordination aspects of the discipline.
\end{abstract}

1 Theoriewildwuchs und Forderung nach wissenschaftlicher Effizienz

Freilich "ist die einschlägige Literatur inzwischen unüberschaubar - und täglich kommt Neues hinzu." (Snell-Hornby et al. 1999: Vorwort). ${ }^{1}$ Die Tatsache, daß diese Klage, in der latent

\footnotetext{
1 Angemerkt sei hier kurz, daß nicht nur der Umfang der übersetzungswissenschaftlichen Literatur ein eignes "Problem" bildet, sondern auch und vielmehr die Form und Gestaltung derselben ein vielsagender Hinweis in bezug auf das Problem, das hier diskutiert werden soll, darstellt. Es gibt sehr wenige Monographien. Stattdessen gibt es Sammelbände, in denen Aufsätze von verschiedenen Autoren unter jeweils bestimmten Themenkreisen vom Herausgeber zusammengefaßt werden. Selbst Bücher, die sich einem Autor verdanken, sind oft, wie im Falle von Stolzes "Übersetzungstheorien", Einführungsbücher, in denen übersetzungswissenschaftlichhistorische Materialien - meist chronologisch oder nach thematischen Schwerpunkten - verarbeitet werden. Also: Nicht nur die Quantität, sondern auch die Vielgestaltigkeit und Mannigfaltigkeit der Ausrichtung dieser
} 
auch ein Aufruf zu einer eventuellen Lösung des Problems mitschwingt, bereits fünf Jahre zurückliegt, könnte Anlaß zu Hoffnungen geben, daß inzwischen wenigstens Schritte in Richtung einer Überschaubarkeit des Feldes getan worden seien. Fünf Jahre, zumal täglich so jedenfalls Snell-Hornbys Überzeugung - neue Ansätze sich zeigen, bedeuten innerhalb der betreffenden Disziplin, d.i. der Übersetzungswissenschaft sehr viel. Zu einem abgewogenen, homogenen, klaren Selbstverständnis einer Disziplin gehört zweifellos die Übersicht über den Stand und Bestand der Forschung und den Wandel dieses Bestandes, kurz, über die vorliegenden Arbeiten und Untersuchungen. Insofern ist sowohl die Feststellung als auch der darin eventuell enthaltene warnende Aufruf legitim, ja sogar gefordert. Andererseits wird, kommt man dieser Forderung nach und bemüht sich darum, über dieses unüberschaubare Feld eine gewisse (durchaus nur annähernde) Übersicht zu erlangen, leicht festzustellen sein, daß die legitime Klage stereotypisch wiederkehrt, sofern sich die Suche nach Klärung und Lichtung freilich nicht vorher in Einzel- und Teilaspekte unlösbar verstrickt hat. Es öffnet sich hier ein Teufelskreis, der das Interesse, in 'übersetzungswissenschaftliche' Untersuchungen und ihren Grundplan einzusehen, bald vernebelt: meist bleibt dieses Interesse in der Klage um die Diffusität und den komplexen Überbau der Disziplin stecken. Diese angesichts des (eigentlich undefinierten und scheinbar undefinierbaren) Wildwuchses einer grenzenlos ausufernden Übersetzungswissenschaft fast nutzlose Klage zu übergehen, das - so der Eindruck - ist inzwischen kaum mehr möglich. So scheint es sich etabliert zu haben, daß in der "einschlägigen Literatur" wohl oder übel auf die Kontroverse um das strittige und bestrittene Selbstverständnis einer ÜW eingegangen werden $m u \beta$, um dann 'zur eigentlichen Sache', d.h. der - oft interdisziplinär angelegten - Beschreibung eines spezifischen ÜProblems selbst zu kommen. Durchaus ließe sich also behaupten, daß diese Art 'Problembezeichnung der Disziplin' zu einem etablierten eigenen 'Untersuchungsschritt' arriviert ist.

Müßte es unter diesen Gesichtspunkten nicht eher verwundern, wenn dieser Pflichtübung des Faches nicht Folge geleistet und von einem problemlosen und intakten Arbeiten der ÜForschung ausgegangen werden würde, d.h., wenn dieses die Fundamente der ÜW angreifende Problem nicht zumindest angeschnitten werden sollte? Diese stereotype Anlage übersetzungswissenschaftlicher Arbeiten deckt zugleich auf, daß sich zunehmend ein Kernproblem verhärtet und kristallisiert, ein Vorgang, der auch die ansteigende Ungeduld nach Lösung desselben Problems impliziert. Die schlußfolgernde Deutung, die übereilten und fast inflationären 'Theorievorschläge' verdankten sich gerade dieser Ungeduld, liegt als Möglichkeit latent in der Luft. Ein unkritischer Zugriff darauf aber wäre seinerseits als eilfertig zu bezeichnen. ${ }^{2}$

\footnotetext{
Quantität dürfte mit berücksichtigt werden, wenn von einer "Unüberschaubarkeit der einschlägigen Literatur" die Rede ist (s. dazu z.B. Stolze 1994: 7f).

2 Warum, wie verschiedentlich beklagt wird, so eine "Vielfalt theoretischer Ansätze" (die zudem bei näherer Auflösung einander oft zu widersprechen scheinen) innerhalb der ÜW sich darbietet, und daß dies unter den heutigen Voraussetzungen durchaus natürlich und logisch (d.i. der Sache angemessen) ist, soll durch diese Überlegungen insgesamt erhellt werden.
} 


\section{Die "Irrelevanz" eines "wahrhaft weiten Feldes"}

Beispiele in bezug auf die oben genannten 'Klagen' anzuführen, gliche einem Eulentragen nach Athen. Dennoch soll dies unternommen werden, denn in den zahllosen "Vorworten" und "Vorbemerkungen" zu übersetzungswissenschaftlichen Büchern, deren Beschaffenheit und Strukturierung selbst einmal eine genaue Analyse verdienen würde (s. dazu Fußnote 1), finden sich vor allem zwei Annäherungen, die diese Klage nicht allein innerdisziplinär (und damit "parteiisch"), sondern allgemein wissenschaftstheoretisch zu fassen versuchen. Einmal spricht Stolze in ihren Übersetzungstheorien von der "Vielfalt der theoretischen Ansätze" (Stolze 1994: 7), die - so ihre Klage - oft aneinander vorbeiredend zu "Verständnisbarrieren beitragen" (Stolze 1994: 7). In dieser Feststellung scheint sich die Interpretation zu bewahrheiten, daß Vielzahl und "Vielfalt" einer wertvollen und seltenen Sache zum Wertverlust derselben führt, also gewissermaßen zur Inflation. Auch wenn diese vielsagende, als Ansatz zu einer grundlegenden Diskussion in bezug auf die Problemstellung selbst sehr hilfreiche Einsicht vor rund zehn Jahren ausgesprochen wurde, gilt sie noch immer und sogar mehr denn je. Ein anderer Ansatz ${ }^{3}$ ist dann schließlich von Brigitte Horn-Helf zur Sprache gebracht worden. Sie wies auf diese inzwischen ubiqitäre Kontroverse in bezug auf das Selbstverständnis einer ÜW hin, indem sie - wiederum an vorhergehende Arbeiten anlehnend ${ }^{4}$ - in ihrem Vorwort zu Technisches Übersetzen in Theorie und Praxis von "Theoriefeindlichkeit der Praxis" einerseits und "Irrelevanz der Wissenschaft" (Horn-Helf 1999: 13) andererseits sprach (womit sie doch wieder sehr anschaulich zugunsten der ÜPraxis Partei ergreift).

Diese beiden wertvollen Ansätze sind aber nicht weiterverfolgt worden, selbst von Stolze ${ }^{5}$ und Horn-Helf nicht. Es blieb beim Ansatz, der - jeweils unterschiedlich graduiert - die Problembezeichnung in nuce enthält. Das Problemerlebnis selbst aber ist gegenwärtig, konkret, ungebrochen grell. Es kann natürlich in diesem Rahmen nicht darum gehen, darüber zu lamentieren, daß eine Chance, die in diesen beiden Ansätzen enthalten ist, ungenützt vertan worden ist. Es geht vielmehr darum, diese Chance noch einmal zu vergegenwärtigen, zu betonen, wachzurufen, um die längst fällige, nötige und klare Problembezeichnung zu ermöglichen. Mit anderen Worten: es geht in dieser Arbeit darum, das unumwundene, nicht von der Hand zu weisende Problemerlebnis innerhalb der "ÜW" genau zu markieren, zu bezeichnen, zu bestimmen. Dabei soll das, was sonst in Vorworten kurz angeschnitten wird,

\footnotetext{
3 'Ansatz' trifft eigentlich den Sachverhalt nicht ganz. Die Horn-Helfsche Interpretation der Problembezeichnung gleicht eher einem ungeduldigen, rigorosen, definitiven Abtun eines Problems, das nur unproduktives Kopfzerbrechen bereitet.

${ }^{4}$ Vor allem in den Begriffen "Kluft", "Brückenschlag" usw. spürt man eine Anlehnung, die aber nicht expliziert wird. Anlehnungen dieser Art sind innerhalb der ÜW-Praxis sehr oft anzutreffen. Dabei macht sich bemerkbar, daß, anstatt daß die Begriffe tiefer und kritisch diskutiert würden, durch die Übernahme bestimmter Wendungen (wie etwa "Kluft") insgeheim das Problem übernommen und weiterverpflanzt wird.

${ }^{5}$ Kürzlich hat Stolze aber in dem Lösungsdenken um eine Standortbestimmung einer Ü-Forschung eine Arbeit vorgelegt, die - wiederum nur und ausschließlich in ihrem allgemein einführenden Einleitungsteil - ganz neue und viel versprechende Aspekte in Aussicht stellt. Diese Aspekte verdienen, gesondert und umfassend durchdacht und ausführlich diskutiert zu werden. Siehe dazu Stolze (2003), im Besonderen S. 24-32.
} 
hier unbeirrt weiter verfolgt werden, d.h., die Problembezeichnung soll selbst problematisiert werden. Eine zufriedenstellende Lösung dessen, was die Problembezeichnung anzielt und zum Gegenstand hat, d.h. des Problemerlebnisses selbst, ist also unter diesen Umständen außer Betracht zu lassen. Denn, es ist ja "ein wahrhaft weites Feld" (Snell-Hornby et al. 1999: Vorwort).

\section{Theoriefähigkeit in bezug auf Teilaspekte}

Die Kompliziertheit der Sache rührt, das muß man in Betracht ziehen, oft von der komplexen Begrifflichkeit, der vielschichtigen Annäherung an die Sache (s. dazu z.B. Stolze 1994: 7f). ${ }^{6}$ Es entsteht durch diese - eigentlich natürliche - Verfeinerung der begrifflichen Apparatur ein nominaler Überbau, der die Sache ins Wesenlose, Unkenntliche verwischt. Geht man den umgekehrten Weg, d.h., löst man sich von jeglicher, die Sache begreifender Apparatur, so bleibt ein Rest an Wesenheit zurück, der sehr nüchtern und klar bezeichnet werden kann. Es ist die Rede von einem Tun, einem Akt, und von der möglichst objektiven Beschreibung desselben. Das ganze Problem rührt also daher, daß sich hinsichtlich des Wegs, dieses Tun möglichst objektiv zu beschreiben, keine Einigung bewerkstelligen lassen will. Die Suche nach diesem möglichst objektiven Weg der Beschreibung des Sachverhaltes (Übersetzung) ist, als Anspruch, legitim, natürlich, konsequent: es geht um Wissenschaftlichkeit. In dieser Setzung, d.h. in dem natürlichen Anspruch, daß die Beschreibung von Übersetzung eine wissenschaftliche Tätigkeit sein müsse, findet die implizite Forderung nach 'möglichst objektiver' Haltung ihren Sinn und ihre gültige Plausibilität. Sie ist in dem Maße gültig, in dem der Wille zur Beschreibung der Sache einem bestimmten Selbstanspruch gehorcht. Die Beschreibung will (und "soll") "wissenschaftlich" sein, um eine begleitende, wertende, feststellende und nicht zuletzt ordnende Reflektion in bezug auf die Sache darstellen zu können.

Die Kennzeichnung der "Wissenschaftlichkeit" ist konkretisiert in einer "Theorie"; erst in der Wesenhaftigkeit der "Theorie" vermag die Beschreibung dem Selbstanspruch (nach Wissenschaftlichkeit) Genüge zu leisten.

"Theoriefeindlichkeit der Praxis" (pragma = Sache) hieße in diesem Sinne dann: Verzicht auf Wissenschaftlichkeit oder zumindest entfiele der Anspruch auf zuverlässige, möglichst objektive Beschreibung. Und parallel dazu bedeutet "Vielfalt theoretischer Ansätze" wiederum 'Unwissenschaftlichkeit', denn, wenn alle möglichen die Sache theoretisierenden Wege dem forschenden Interesse offen stünden, müßte alle Beschreibung der Sache als 'relativ objektiv' erscheinen. Das konkrete Erlebnis des Problems innerhalb einer

\footnotetext{
6 Stolze spricht hier von "Uneinheitlichkeit im Begriffsapparat". Daß diese von der ungeordneten und unkoordinierten interdisziplinären Struktur der ÜW herrührt, scheint ebenso klar und leicht nachzuvollziehen zu sein. Dennoch ist die dringende Aufgabe, eine Ordnung und Lichtung in dieselbe Begriffsapparatur zu bringen, noch immer nicht wahrgenommen worden. Dazu wäre geboten, zunächst die Interdisziplinarität, wie sie sich innerhalb der ÜW darbietet, genau und im Rahmen allgemeiner wissenschaftstheoretischer Überlegungen zu kennzeichnen, zu verstehen und Prioritäten zu setzen, d.h., alle in die ÜW eingreifenden Disziplinen in ihrer diesbezüglichen Wirkungsbreite untereinander abzuwiegen und einzuordnen. Hierzu wären u.a. Holmes Überlegungen in Bezug auf die "Feldtheorie" ein sehr hilfreicher Ansatz zur betreffenden Diskussion (s. dazu Stolze 1994: 131).
} 
übersetzungswissenschaftlichen Untersuchung betrifft also die Grundmauern und die Basis der Untersuchung selbst. Es besteht nicht nur die Freiheit, beliebig an die Sache herangehen zu können, sondern dieselbe Freiheit ist zugleich Erfordernis (da es die jeweils einmalige Sache zu erfordern scheint). Dieser Punkt allein wäre aber nicht von so entscheidendem Belang, im Gegenteil, denn mögliche und legitime Mannigfaltigkeit der Untersuchungsmethoden könnte auch auf eine ausgebaute Forschung und Herrschaft der disziplinären Apparatur über die Sache deuten. Das Problem liegt tiefer respektive eigentlich auf der Oberfläche, nämlich bei der 'Sache' selbst. Kurz, in dem Begriff "Sache". Mit anderen Worten: Die Schwierigkeit, die Sache auf möglichst objektiven Wege zu beschreiben, liegt nicht darin begründet, daß ein solcher verlässlicher Weg nicht gefunden wird, sondern in der Freiheit, die Sache jedes mal in einem spezifischen Licht (und somit jeweils als etwas Anderes, Teilaspektuelles) begreifen zu können (s. dazu den Begriff "Teilaspekte" z.B. bei Stolze 1994: 7), also in dem jeweiligen Begreifen der Sache selbst.

Theoriefeindlichkeit der Praxis (als Wirklichkeitsbezug der Sache) ist - das wird oft unterschlagen - nicht allein in bezug auf übersetzungswissenschaftliche Forschung gegeben; ganz allgemein lässt sich feststellen, daß Theorien und wissenschaftlich fundierte Modelle eine verkürzende Vergröberung der Sache darstellen, auch wenn die Sache einen durch Struktur und sinnliche Anschaulichkeit begrenzten Gegenstand darstellt und nicht - wie im Falle der Übersetzung - einem durch Faktoren und Teilprozesse dynamisch gekennzeichneten Tun, Handeln oder Akt unterliegt. Die möglichst umfassende und objektive Erfassung einer Sache, in der - wie in der ÜW - ein menschliches Tun als Bestandteil der zu erfassenden totalen Sache enthalten ist, wird notwendigerweise außerdem noch erschwert werden, und zwar dadurch, daß die Sache selbst als eine Einheit, als Totales, Festes nicht eindeutig erkannt (oder beschrieben) werden kann. Mit anderen Worten: Je nach dem, was für ein Teilbegriff von der Sache als ausschlaggebend angenommen wird, wird eine Argumentation in bezug auf denselben Teilaspekt der Sache entsprechende Erkenntnisse herausarbeiten müssen. Wenn also der Begriff "Theorie" den Anspruch erhebt, umfassend, möglichst objektiv, die Sache verallgemeinernd, kurz 'grundlegend' zu sein (vgl. dazu Reiß/Vermeer 1991), so ist es in bezug auf "Übersetzung" als einer komplexen Sache natürlich, daß erstens sich viele Theorien anhäufen, zweitens ist es aber gerade aufgrund dieser Möglichkeit der Theorienvielfalt logisch, daß diese möglichen Theorien einander aufheben an Aussagekraft, d.h., daß eine Theoriefeindlichkeit der Praxis (des Wirklichkeitsbezuges der Sache) sich zusehends spürbar macht. Unter diesen allgemeinen Forschungskonstellationen scheint es einerseits konsequent, daß sich viele Theorien in bezug auf die 'Sache' anbieten, aber andererseits gerade dadurch, daß viele und mannigfaltige theoretische Ansätze möglich werden, wiederum unvermeidlich, daß diese in ihrer Wirksamkeit und umfassenden Aussagekraft sich gegenseitig aufheben.

Von diesen allgemeinen Überlegungen nun einen konkreten, anschaulichen Totalblick auf das Problemfeld herauszuarbeiten, ohne in eine terminologische Verwicklung zu geraten, wird nicht mehr schwer fallen. 
Die übersetzungswissenschaftliche Forschung ist gekennzeichnet durch Mannigfaltigkeit der Theorien. Alle Theorien mögen - das sei nun als Annahme einmal gegeben - in sich durchaus schlüssig sein. Aber dadurch, daß - wie in der Klage Stolzes latent enthalten - diese Mannigfaltigkeit an Theorien möglich ist, gerade wegen der Vielfalt, hinken dieselben Theorien der Praxis hinterher. So entsteht eine Theoriefeindlichkeit der Praxis, diese aber drückt sich in der "Irrelevanz der Wissenschaftlichkeit" aus. ${ }^{7}$ So etwa könnten die Hauptargumente auf einen Nenner gebracht, als solches aber auch im Gesamtblick näher erhellt werden. Die Begründung dieses Gesamtbildes liegt im Sosein der Sache, d.h. dem komplexen, vielschichtigen Wirklichkeitsbezug der Sache, dementsprechend auch in der etablierten Forschungspraxis. In dieser ist die Sache - wie oben bereits angedeutet - jedes mal in einem "Teilaspekt" begriffen und aufgefasst, und die Theorien richten sich nach diesen Teilaspekten (s. dazu z.B. Stolze 1994: 7). ${ }^{8}$ Die Notwendigkeit einer Konzentration der Diskussion auf Begriffe wie "Teiltheorien" oder "komplexe Theorien" oder überhaupt auf die Fähigkeit der ÜW, komplexe, d.i. übersetzungswissenschaftliche Theorien bilden zu können, dürfte auf der Hand liegen. In diesem Rahmen bewegt sich nun die allgemeine Problembezeichnung, d.h. die Bestimmung des Problems in bezug auf die Selbstdefinition und des Standortes einer ÜW.

An dieser Stelle liegt es nahe zu fragen, was im Rahmen der übersetzungsorientierten Forschung unter dem Begriff "Theorien" verstanden wird. Denn, wenn Theorien gegeben sind und die diesbezügliche Wissenschaft dennoch als irrelevant aufgefasst wird, so dürfte eines dieser Argumentationspunkte ein Problem bilden, mit anderen Worten: entweder sind die Theorien nicht stichhaltig oder die Wissenschaft besitzt sehr wohl Relevanz. Die vielseitigen Klagen in bezug auf die Schwierigkeiten einer Standortbestimmung der ÜW weisen eher auf die erste Behauptung hin. Andererseits besteht Einigkeit darin, daß die ÜW in den letzten Jahren immer weiter floriert, der Begriff 'Wildwuchs' liegt - wie oben bereits angemerkt - in der Luft, greifbar nahe und wird wohl bald beschwert werden müssen. Auch hier wieder stellt sich der Eindruck ein, daß im Zusammenhang mit der Problembestimmung und -bezeichnung irgendetwas nicht ganz stimmen kann. Hier liegt ein - unproduktiver - Widerspruch in der Bestimmung der Sache und der Problematik: eine als irrelevant erkannte Tätigkeit unbedingt weiterzuverfolgen und zu forcieren, deutet auf Goethes Wort von der "unbedingten Tätigkeit", die "schnurstracks in den Bankerott" führt.

\footnotetext{
7 Übrigens enthält Horn-Helfs Bestimmung in bezug auf die "Irrelevanz der Wissenschaft" dieselbe Konkretisierung wie "Theoriefeindlichkeit der Praxis"; es geht also nicht um einen "einerseits-andererseits", sondern um einen "sowohl-als auch"-Bezug. Wenn man noch genauer hinsieht, so wird selbst eine sowohl-als auchBestimmung aufgehoben, denn diese deutet auf zwei unterschiedliche Wesenheiten. Beides bildet in der Konkretisierung denselben Sachverhalt. Das heißt: Die Theoriefeindlichkeit der Praxis drückt sich in der Irrelevanz der Wissenschaft aus. So weit kann es kommen, wenn man sich von der Anschaulichkeit der Sache trennt und - wie es die in der übersetzungswissenschaftlichen Forschung etablierte Praxis offenlegt - lediglich der Terminologie der Sache dient.

8 Stolze unterstreicht an der genannten Stelle, daß "in den einzelnen Theorien ... jeweils ein Gedanke besonders hervorgehoben" wird, und geht damit vom umgekehrten Standpunkt aus.
} 
Aus all diesen Überlegungen wird ersichtlich, daß die etablierte und akzeptierte Problembezeichnung viele Lücken und vage Stellen enthält; es tut also Not, die Problembezeichnung genauer und konsequenter $\mathrm{zu}$ verfolgen. Dazu ist geboten, unvoreingenommen und konkret auf die Sache selbst zu sehen. Am Ende wäre auch zu entscheiden, ob die Klagen wirklich begründet und berechtigt sind, oder ob das Problem, als konkretes Erlebnis innerhalb der Forschungspraxis, woanders zu suchen wäre.

\section{$4 \quad$ Nominales und anschauliches Denken in der Ü-Forschung}

Ganz abgesehen davon, ob die überall wiederholte Markierung des Problems begründet ist oder nicht und woher nun diese rührt, fällt im allgemeinen Grundton der Debatte um die Selbstbestimmung der ÜW folgendes auf: Übersetzung sei eine "Handlung", eine Fertigkeit, ein Akt, ein Verhalten, also eine praxisorientierte, immens wandlungsfähige und -reiche Sache, und Wissenschaft sei allgemein auf modellierende, d.h. die Sache verallgemeinernde Theorien ausgerichtet. Und die strittige Frage eben bestehe darin, daß "Übersetzung" (in jedem Übersetzungsfall jeweils spezifisch und wandlungsreich) sich nicht (oder, nach konträrem Standpunkt: sehr wohl) mit "Wissenschaft" (modellierendes Beschreiben und Erfassen) problemlos vereinigen lassen könne. Horn-Helfs Hinweise dürften sich diesen Argumenten verdanken. In diesem begrifflichen Widerspruch kommt folgende Überlegung zum Ausdruck: Die "ÜW", d.h. also die Zusammensetzung der Begriffe "Übersetzung" und "Wissenschaft", stellt ein - wiederum wissenschaftliches - Problem dar, das sich nicht lösen lassen will. Bei unvoreingenommener Annäherung an dieses verwickelte Geflecht von Problemen kommen einem tiefe Bedenken, die, ist man darin verstrickt, schwerlich wahrgenommen werden. Der Sachverhalt, einmal ganz konkret und fasslich, nüchtern veranschaulicht, zwingt dazu, sich einmal für kurz von jeder voreingenommenen, vorgedeuteten Terminologie zu lösen:

Eine "Theoriefeindlichkeit der Praxis" (die Übersetzung betreffend) hat zur Konsequenz, daß keine Theorie in bezug auf den Gegenstand und die Praxis Gültigkeit besitzt, darüberhinaus aber und noch viel mehr, daß gar nichts behauptet werden kann. In diesem Fall würde nämlich jede Behauptung richtig und legitim sein, was dazu führte, daß keine Behauptung wahr, oder richtiger ausgedrückt: stichhaltig wäre. Damit wäre aber zugleich die "Irrelevanz der Wissenschaft" belegt und begründet. Die beiden das Problem in seinem Widerspruch bezeichnenden Begriffe erweisen sich unter diesem Blickpunkt also - wie vorher angenommen und propagiert - nicht als kontroverse Argumentspunkte, die zum Widerspruch führen, sondern nur als die Konsequenz des einen aus dem anderen. ${ }^{9}$ Wieder wird ersichtlich, daß die Problembezeichnung selbst noch einen enormen Denkprozeß zu durchlaufen hat, ehe das Problem selbst wird erkannt werden können. Hierbei scheint vor allem eine bestimmte

\footnotetext{
${ }^{9}$ Um nur diesen an sich unerheblichen Punkt zu korrigieren wäre der Begriff "Praxisscheu der Wissenschaft" vielleicht angebracht. Aber selbst dieser Begriff impliziert wiederum etliche Fanggruben, so daß man versucht ist, sich aus dieser fruchtlosen Diskussion ganz herauszuhalten.
} 
Haltung den Blick gefangen zu halten, und zwar die durchgängig 'nominale'10 Struktur der übersetzungswissenschaftlichen Argumentationsweise, die zu ersetzen wäre durch ein auf Anschaulichkeit beruhendes, allgemein wissenschaftstheoretischen Erfordernissen gehorchendes Begreifen der Grundprobleme sowie der Sache selbst.

Eine Durchsicht der Konsequenzen, die sich aus den oben markierten 'Kontrapunkten'11 ergeben, müßte zugleich offenlegen, wie Problembezeichnung und Problemerlebnis in eines gehen. Wenn beispielsweise die "Irrelevanz der Wissenschaft" stichhaltig wäre (was sie - wie wir vordergründig erkannt haben - ja durchaus sein kann), so wäre jede Behauptung über die Übersetzung wahr, so hatten wir oben gefolgert, aber die Konsequenz aus dieser Folgerung in bezug auf die Wissenschaftspraxis wäre reine Beliebigkeit des Vorgehens und Willkürlichkeit der Aussagen. Mit dieser Konsequenz wäre schließlich jeder Schritt unmöglich gemacht, d.h. wissenschaftstheoretisch gesehen unmöglich, was die Praxis selbst angeht, mag in diesem Rahmen dahin gestellt sein. Die Konsequenz aus der anderen Argumentation, nämlich Praxisferne und -scheu der Wissenschaft, müßte ihrerseits ein andres Faktum offenlegen, und zwar daß ihre Aussagen von der Sache wegtendierten, haltlos und wie in der Luft hingen, d.h., zusehends sich in eine reine nominale Sichtweise verflüchtigten, mit anderen Worten, jeder Anschaulichkeit entbehrten. Zusammenfassend ließe sich also sagen, daß in dem Falle, daß die Problembezeichnung stichhaltig wäre, eine Ü-Wissenschaft zum einen willkürliche Untersuchungsschritte und Beliebigkeit der Ergebnisse, zum andern aber eine dem Gegenstand nur mittelbar verbundene nominale Denkstruktur aufweisen müßte.

Interessant ist, daß durch eine ganz allgemeine Durchsicht der bisherigen Ü-Forschung ${ }^{12}$ diese konstruierten Sachverhalte bestätigt werden; daß sich also im Gefolge eines nominal

\footnotetext{
${ }^{10}$ Diese Überlegung verdient, gesondert und mit konkreten Beispielen belegt und differenziert zu werden. Hier sei einstweilen angemerkt, daß darunter folgendes verstanden wird: Die Sichtweise, in der sich das übersetzungswissenschaftliche Forschungsinteresse artikuliert, beruft sich nicht unmittelbar auf den Gegenstandsbereich, sondern eher auf einen - meist fremddisziplinär begründeten - Begriff oder terminologischen Apparat in bezug auf (vermeintlich) denselben Gegenstandsbereich. Ein interessantes Beispiel in diesem Zusammenhang stellt der in der Ü-Forschung ohne jede Differenzierung etablierte (Haupt-)Begriff "Ausgangstext" dar. AT wird implizit als immer etwas Bestimmtes aufgefasst, ohne daß explizit gemacht würde, daß im je spezifischen Untersuchungsfall derselbe Gegenstand variiert, geschweige denn, daß man dessen einsichtig würde, daß AT in seltensten Fällen tatsächlich "Ausgang" (für was auch immer) bildet. So geistert innerhalb der Ü-Forschung das 'nominale Phänomen' eines AT noch immer herum, ohne daß bisher Zweifel entstanden wären, die durch eine anschauliche Vermittelung des Gegenstands längst sich sehr wohl aufgedrängt haben müßten.

${ }^{11}$ Hier wäre noch einmal zu betonen, daß es sich nicht um ein Unikum handelt, daß also Horn-Helf nicht alleine dasteht mit ihrer Problembezeichnung, sondern daß die Diskrepanz zwischen Ü-Theorie und Ü-Praxis (um es noch allgemeiner und neutral zu bezeichnen) eine nicht mehr wegzudenkende Haltung der "Ü-Wissenschaft" selbst geworden ist. Deshalb scheint es unnötig, eine Quelle gesondert anzugeben; man lese in irgendeine Buchveröffentlichung oder in einen Aufsatz, so wird man als einleitende Worte jedes Mal, anders gedeutet, zum nämlichen Problem Ausführungen und Vermerke finden.

${ }^{12}$ Hierzu und daß bereits dies ein fast unüberwindbar problematisches Unternehmen ist, siehe im weiteren die folgenden Gedanken- und Diskussionsschritte. Der Ü-Forscher, der sich diesen Fragen und Problemen ernstlich stellt, wird sich fast in die Rolle der Sysiphos-Figur versetzt fühlen: er rollt seinen Fels hinauf auf den Hügel, wohl wissend, daß derselbe wieder nur runterrollen wird, und die Arbeit wird wieder - und das bis ins Endlose erneut und von vorne in Angriff genommen werden müssen. So jedenfalls steht es im Mythos.
} 
orientierten Denkens eine Problembezeichnung, die sich selbst als problematisch erweist, unmittelbar in der Wissenschaftspraxis als ein die Anschauung verneinendes Problemerlebnis auswirkt und darstellt. Zumindest scheint also die Verstrickung zwischen Problemdefinition und Problemkonkretisation selbst stichhaltig und nachvollziehbar zu sein. Noch interessanter wäre, der Frage nachzugehen, ob das Problemerlebnis aus der Problembezeichnung resultiert oder ob nun ein Problem innerhalb der Ü-Forschung zu der (an sich selbst schon problematischen) Problembezeichnung nötigt? Im Falle, daß die ganze ü-orientierte Forschung einem grundlegenden Problem nur deshalb unterliegt, weil die (irreführende, weil die Anschaulichkeit ausgrenzende, nominale) Problembezeichnung dies aufdrängt, so wäre es eine Frage der Eigendisziplin; d.h., irgendein heterogenes, etwa terminologisch gedachtes Gewicht würde als "Fremdkörper" innerhalb der ÜW auf die Forschungspraxis hemmend einwirken. Und die Lösung wäre mit dieser Bezeichnung bereits angedeutet, zumindest die Stelle, wo sich eine Lösung finden lassen könnte. Im Falle aber, daß innerhalb der ÜW sich bestimmte, konkrete, der "reinen" Wissenschaftspraxis widersprechende Hürden und Hindernisse ergeben, welche zu der bereits genannten Problembezeichnung zwingen, müßte man sich grundlegende Gedanken zu dem gegebenen Forschungsgegenstand an sich machen, und zwar anlehnend an grundlegende wissenschaftstheoretische Hauptkriterien, mit der Absicht, herauszubekommen, ob dieser Gegenstand ("Übersetzung") wissenschaftlich erkannt und präzise beschrieben werden könne oder ob er sich der wissenschaftlichen Untersuchung entziehe. Im ersten Fall hätte man eine Problemstellung vor sich, die wir hier ganz allgemein als Eigendisziplinarität bezeichnen wollen, im andern Fall wäre es eine Wissenschaftstheorie (in die der problematische Begriff der spezifischen Interdisziplinarität innerhalb der ÜW hineinreicht), die wir primär zu durchleuchten hätten. Doch in beiden Fällen ist geboten, das ganze Feld, von welchem Standpunkt auch immer, zu durchleuchten, kritisch zu hinterfragen. Und das ist eine fast endlose, zumal aussichtslose Arbeit, gleich der des Sysiphos', angesichts des ausgiebigen Materials, das sich einem vor den Augen dartut; und: "täglich kommt Neues hinzu".

Aus all diesen Gesichtspunkten und Überlegungen wird eines klar, und zwar, daß in der zentralen Klage ein problematischer, bisher nicht zur Genüge reflektierter Anspruch liegt, der sie motiviert, eine Deutung, ein 'Vor-Urteil' in bezug auf das, was man "Wissenschaftlichkeit" nennt. Wissenschaft besitzt den Anspruch, 'objektive Wahrheiten' über den von ihr untersuchten Gegenstand zu modellieren, zu erschließen. Es geht - als Überbau und institutionelles Vorgehen - dabei um totale Erfassung der Sache, ganzheitliche Klärung eines komplexen Wirklichkeitsbezugs. Doch bei der Übersetzung ist diese Erfassung nicht so einfach und klar, da die Praxis der Übersetzung - wie oben bereits erwähnt - die Reflektion über das Übersetzen stets überholt. Das Reflektieren und das Tun in bezug auf Übersetzung sind - im Übersetzungsvorgang - eigentlich nie voneinander zu trennen, es liegt in der Natur der Übersetzung, $\mathrm{da}$ Tun und Reflektion in bezug auf das eigne Tun sich gleichzeitig realisieren. Nur im 'wissenschaftlichen Rahmen' entsteht die Diskrepanz zwischen Tun und Reflektion, da das Tun ausgeschaltet wird und selbst als scheinbar objektiv erfassbares Forschungsziel dient. Die Theoriefeindlichkeit der (Ü-)Praxis ist also nichts andres als natürlicher, impliziter Theorieüberfluß einer Praxis, die sich - nur im künstlichen Rahmen der 'Wissenschaftlichkeit' - der 
Selbstreflektion entledigt hat. Dieselbe Wissenschaftlichkeit aber geht von typisierenden und kategorisierenden Übersetzungsfällen aus, die es in der Praxis kaum gibt. Anders als typisierend und kategorisierend vermag aber eine Wissenschaft den betreffenden Gegenstand nicht zu erfassen.

\section{$5 \quad$ Zwischenbilanz und Folgerungen}

Die Bestimmung und Festlegung der Sache und dessen, was man unter dieser begrifflichen Festlegung versteht, scheint ein größeres Problem zu bilden als die weitere, gegenstandsbezogene Untersuchung, die ja - sieht man genauer hin - der vorhergehenden Bestimmung dessen, was "Übersetzung" ist, konsequent Folge leistet. Die Untersuchungen selbst als forschungstechnisches Vorgehen stellen also (zunächst) kein Problem dar, denn das, was durch die vielfältigen Untersuchungen in bezug auf das Wesen der Übersetzung erkannt wird, scheint plausibel, in sich schlüssig und doch irgendwie "wahr" zu sein. ${ }^{13}$ Wo liegt also das Problemerlebnis? Das Problemerlebnis liegt in der problematischen, anspruchsvollen Problembezeichnung verborgen. Die ÜW sucht nach ihrem Problem, das ist ihr Problem. ${ }^{14}$ Es handelt sich um ein rein wissenschaftstheoretisches Problem, um einen selbstreflektierenden, auf das verstehende Verfahren selbst zurückleuchtenden Apparat.

Diese Problemdiskussion ist - dies dürfte spürbar geworden sein - für niemanden zufriedenstellend. Irgendwie entsteht dabei auch das Gefühl, daß an der konkreten Problematik vorbeigeredet würde, d.h., je mehr terminologisch untermauerte, rein begriffliche Argumentation zur Sache herangetragen wird, umso mehr entfernt man sich vom Kernpunkt der Sache. Längst hätte ein tiefer Zweifel inbezug auf diese Apparatur selbst entstehen müssen. ${ }^{15}$ Beispielsweise liegen bereits in dem spezifischen Gebrauch des Begriffes "Theorie" seitens der ÜW so viele Fanggruben und Dunkelheiten, daß man geneigt ist, den Argumenten (Theoriefeindlichkeit der Ü-Praxis, Vielfalt der Theorien usw.) - aber nur unter der Bedingung, daß der Begriff selbst (Theorie) grundlegend in seiner ü-spezifischen Bedeutung geklärt wird - Recht zu geben. Ebenso verhält es sich mit vielen anderen Hauptbegriffen wie etwa "AT"; "ZT"; "Text" (in bezug auf Übersetzungswissenschaft); "Translation"; "Transfer"; "ÜMethode", "Äquivalenz"16 usw. Ebenso müßte die etablierte Forschungstechnik sowie die Argumentationskette in der Ü-Forschung kritisch durchdiskutiert werden in bezug auf ihre

\footnotetext{
13 Meist handelt es sich um die Deskription von Einzelerscheinungen innerhalb des Ü-Vorgangs, weshalb die Bezeichnung "wahr" dem Sachverhalt nicht ganz entgegenkommt. Deshalb die Hervorhebung durch Anführungszeichen.

14 Stolze spricht in Hermeneutik und Translation von einem "präparadigmatischen Zustand", in der die Translationswissenschaft sich befinde (Stolze 2003: 28). Dieser sehr nüchterne Ansatz dürfte - wird er ernst genommen und weiter durchdacht - innerhalb des Werdeprozesses einer sich selbst behauptenden ÜW eine entscheidende Bedeutung haben.

15 Diese Zweifel scheinen Stolze in ihren einleitenden Überlegungen zu Übersetzungstheorien zu leiten. An dieser Stelle sei, auch wenn es in diesem Rahmen verwundern sollte, eine Analogie zu diesem Sachverhalt angeboten: dieser ganze Sachverhalt in bezug auf die Erfassung des Problems selbst erinnert an die Konstellation in der Matrix-Welt. Man muss sich schon in sie begeben, um ihr beizukommen, vom Raumschiff allein aus vermag man sie nicht zu beeinflussen. Dabei weiß man, daß man es mit einer Fiktion, einem Trugbild zu tun hat.

16 Dieser Begriff unterliegt inzwischen einem Bedeutungswandel, wenn nicht sogar einem Bedeutungsschwund.
} 
Stichhaltigkeit. In diesem Zusammenhang könnte eine Skizze der grundlegenden, "übersetzungwissenschaftlichen" Argumentationshaltung sehr aufschlußreich sein, wobei auch und vor allem zum Ausdruck kommen sollte, mit welcher impliziten Bedeutung der Begriff "Theorie" innerhalb der Ü-Forschung gehandhabt wird. So basiert die gängige Ü-Forschung großteils auf drei grundlegenden Schritten, die sich implizit abzeichnen, nämlich:

\section{Schritt:}

Definition des Begriffs "Übersetzung" (Translation) in Form von: "Übersetzen/Übersetzung/ Translation ist $x y z$ ". Diesen ersten Schritt motiviert und leitet wiederum eine heimliche Implikation. Es wird oft eine bestimmte Kategorie von Übersetzung primär gegen etwaige andere Kategorien ausgespielt, d.h., als ausschlaggebend für die 'grundlegende' allgemeine Determinierung von Übersetzung schlechthin gedacht, beispielsweise die literarische Übersetzung gegen das technische Übersetzen usw. Andererseits liegt seit längerem, d.h. mit dem komplexeren Ausbau der ÜW, einem solchen Defintionsgebaren mehr und mehr die Intention zugrunde, die Übersetzung als Ganzes, d.h. als ein Verfahren zu verstehen, anstatt wie früher das Augenmerk auf das "Resultat" zu konzentrieren. Diese Tendenz, diese Verlagerung des Forschungsinteresses deutet ihrerseits auf die Problematik hin: Wo setzt man bei der Definition an? Was soll definiert werden?

\section{Schritt:}

\section{Begründung dieser Definition auf jeweils unterschiedlich ausgerichtetem interdisziplinären} Wege. D.h., es findet eine Adaption von Fremdtheorien, quasi als 'Importgüter', auf übersetzungswissenschaftliche Fragen statt, wobei oft eine Vermengung diverser Theorien zu einer Einheit, in Form von einer "komplexen Theorie", vollzogen wird. Dieser Gedanke ist im Grunde nicht neu. Beispielsweise sieht man bereits bei Vermeer die handlungstheoretisch orientierte Kommunikationswissenschaft mit Kulturwissenschaft vermengt, aber als eine "Translationstheorie" ausgedrückt. In vielen anderen "übersetzungstheoretischen Modellen" sind es Variationen von linguistischen oder textlinguistischen Theorien (s. dazu z.B. Schäffner 2000). Eine allgemeine Durchsicht der bisher dargebotenen "Theorien" auf die Frage hin, in welchem Grad eine Komplexität von Theorien darin impliziert ist, wäre unter diesem Gesichtspunkt also unbedingt zu erarbeiten. Diese Arbeit, die an sich schon ein immenses Forschungsvolumen bildet, wäre nur eine Vorarbeit zu nennen, eine Voraussetzung, die es ermöglichte, den Blick zu schärfen für das interne Forschungsproblem der ÜW schlechthin.

\section{Schritt:}

Beweisführung (d.h. Deskription) vermittels eines (konstruierten oder realisierten) $\ddot{U}$ Falles, um die vorhergehenden Definitionsschritte zu begründen. Hier wird konkret die (normativ begründete) Ü-Methode diskutiert und anhand des Beispiels veranschaulicht. ${ }^{17}$

$17 \mathrm{Zu}$ beachten wäre in diesem Zusammenhang, daß durchaus eine "Praxisscheu" der wissenschaftlichen Forschung spürbar ist, daß also der dritte Schritt auch entfallen kann, denn durch die Beweisführung anhand 
Dabei kann es sich auch um übersetzetzungskritische Gesichtspunkte handeln; vorhergehende Konklusionen können an einem konstruierten (ü-methodische Untersuchung) oder realisierten (ü-kritische Untersuchung) Beispiel belegt und bewiesen werden, an dem endgültigen 'Erkenntniswert' der gesamten Untersuchung wird sich nichts ändern.

Dieses inzwischen festgefahrene Argumentationsmuster und Forschungsgebaren wäre natürlich und unbedingt - und nicht nur in bezug auf seine Stichhaltigkeit in ÜWUntersuchungen, sondern allgemein wissenschaftstheoretisch - zu hinterfragen. Schon hier zeichnet sich ab, daß die Ü-Forschung eine in ihren Grenzen nicht klar definierte Disziplin ist, sondern eine von verschiedenen Disziplinen zusammengesetzte, in sich unbestimmte und dynamische Agglomeration von Forschungsmustern darstellt, die den Anspruch stellt, eine (eigenständige oder interdisziplinäre) Disziplin zu sein. Selbst im Falle der Akzeptanz einer Interdisziplinarität müßte diese, d.h. die interdisziplinäre Forschungspalette, genau begrenzt und bestimmt werden.

"Ü-Theorien" scheinen also - unter diesen Gesichtspunkten betrachtet - im Allgemeinen nur ü-methodische Modelle oder Vorschläge zu solchen ü-methodischen Richtlinien zu bilden. Vereinfacht könnte man sagen: Eine Ü-Theorie stellt den Anspruch, die Ü-Praxis durch methodische Konzentrierung zu 'umgarnen', um ihr beizukommen, sie in ihrer vielgestaltigen und unbestimmbaren Komplexität zu normieren. Man ist versucht zu fragen, was daran falsch oder illegitim sei. Zunächst scheint diese Zielgerichtetheit, dieser Anspruch sehr plausibel, ja fast logisch, aber nur fast. Denn wenn man sich wieder an die allgemeine Argumentation erinnert, so verschiebt sich zunächst die Bedeutung von "Theoriefeindlichkeit der Ü-Praxis". Es handelt sich nicht eigentlich um eine "Theoriefeindlichkeit der Ü-Praxis", sondern doch um eine "Praxisscheu der Ü-Theorie". ${ }^{18}$ Doch das wäre nicht das eigentliche Dilemma, denn diese Bedeutungsverlagerung ändert den Sachverhalt nicht, nur die Betonung wird - je nach Standpunkt - verschoben, oder eigentlicher: umgekehrt. Durchaus, ein Erkenntnisgewinn mag in diesem Ansatz stecken, doch die Sache liegt weiterhin im Dunkeln. Wenn man aber dazu noch den Begriff "Theorie" in seinem ü-forschungsspezifischen Gebrauch, und zwar als ümethodische Grundlegung versteht, so wird die Sache klarer: Die Ü-Praxis darf also durchaus den Ü-Theorien feindlich gegenüberstehen (oder umgekehrt, d.h., die Ü-Theorie muß unter diesem Gesichtspunkt vor der Ü-Praxis scheitern), da diese nur eine in sie hineinwirkende Methode ausmachen will. Die Ü-Praxis enthält nämlich unüberschaubar zahlreiche und mannigfaltige Möglichkeiten zu einer, und zwar nur zu einer in dem betreffenden Ü-Fall spezifischen Methode. Und in diesem Zusammenhang ist es genauso natürlich, daß eine Vielfalt von Theorien sich anbietet, solange man unter Theorien nur ü-methodische Vorschläge versteht. Denn jeder Ü-Fall impliziert im Grunde eine eigne, einmalige, spezifische "Ü-Theorie", die mit keiner anderen zu vergleichen ist. Insofern kann am Ende beiden Argumenten (Theoriefeindlichkeit der Ü-Praxis; Vielfalt der Theorien) zugestimmt

eines Ü-Beispiels begibt man sich oft in ein gewagtes Terrain, wo die vorhergehenden Konklusionen sehr wohl und unerwarteterweise widerlegt werden können.

18 Dieser Sachverhalt dürfte, gesondert und vertieft in einer eignen Studie behandelt, zu grundlegenden und fruchtbaren Erkenntnissen Anlaß geben. 
werden, solange natürlich der Begriff "Theorie" in seiner ü-wissenschaftlichen, verdeckten Bedeutung als übersetzungsmethodische Vorschläge verstanden wird. Durch die gegebene Argumentation wird also klar, daß die darin liegende Erkenntnis des Sachverhalts legitim und gerechtfertigt ist; ein solcher Problemfall, von dem Stolze und Horn-Helf auszugehen scheinen, ist also durchaus existent. Nur die Klage ist seltsam; mit der Klage, daß ein solcher Problemfall besteht, kommt man nicht zu Rande. Denn die Natur des Übersetzens bringt es mit sich, daß nicht bestimmte, festlegbare Methoden sie bestimmen, sondern, daß viele und jeweils variierende Methoden, d.h. in übersetzungswissenschaftlicher Terminologie: "ÜTheorien", in ihr stets latent mitgegeben sind. Der beklagende Ton in der Argumentation ist also vordergründig unberechtigt. Und hintergründig? Hintergründig liegt in der Klage der implizite Anspruch nach "reinen Theorien", also wissenschaftstheoretisch untermauerten Theorien. Die ganze Klage ist also zugleich Beleg und Veranschaulichung des Problems selbst: Man bewegt sich hintergründig im Rahmen einer klaren, allgemein etablierten Wissenschaftlichkeit, vordergründig versucht man auf übersetzungswissenschaftlichem, d.h. provisorischem Terrain im Dunkeln sich zurechtzufinden. Beides, d.h. Kriterien traditioneller Wissenschaftspraxis und "ÜW" lassen sich schwer vereinbaren.

\section{Schluß und Konklusion}

Statt Klage, die ja berechtigt ist, aber nichts fruchtet, außer daß die Debatte weiter auf fruchtlosem Wege fortgeführt wird und schließlich durch oftmalige Wiederholung zu Lähmungserscheinungen Anlaß gibt, statt ambivalente Klage also ist klare Selbstorientierung und Standortbestimmung geboten. Bei dieser Standortbestimmung kann es aber nicht darum gehen, die einmal etablierten Grenzen allgemeiner Wissenschaftlichkeit so zu verschieben (beispielsweise durch begriffliche Ansätze wie "angewandte Wissenschaft", vgl. GerzymischArbogast (1994), übrigens: ein contradictio in adiecto), nur um auch die ÜW unter Dach und Fach bringen zu können. Und genauso trägt die Haltung, die ÜW allgemein als "Interdisziplin" abzutun und nicht genau zu beschreiben, was forschungstechnisch darunter zu verstehen ist, nur dazu bei, das Problem zu verharmlosen und dadurch erst recht eigentlich unnötige, zusätzliche Verwicklung zu erzeugen oder zumindest möglich zu machen.

Bei der Selbstbestimmung einer Wissenschaft sind grundlegende, wissenschaftstheoretisch reine Begriffe zunächst von großem Wert. Insofern sei hier in bezug auf die Problembezeichnung der übersetzungswissenschaftlichen Forschung folgendes Begriffspaar zur Diskussion gestellt: Subordinanz und Koordinanz ${ }^{19}$ (s. dazu Schopenhauer 1977: 100-

\footnotetext{
19 Auch Stolze beruft sich in Hermeneutik und Translation (2003: 29), gerade im Zusammenhang mit der Problematik der Standortbestimmung der ÜW, auf die Begriffe "Koordinaten" und "Subordinaten". Doch müsste genauer der Unterschied der Interpretation dieser Begriffe oder eigentlicher ihrer Bedeutung im Rahmen der grundlegenden Ü-Forschungsprobleme markiert werden, was oben ansatzweise erfolgen soll. Dabei wäre folgende Überlegung vor Augen zu halten: Dieses Begriffspaar ist eine Anlehnung an Schopenhauers Ansatz. Schopenhauer spricht in bezug auf das taugliche Wirken und Wesen jeglicher intakten Wissenschaftlichkeit von den Prinzipien der "Subordination" und "Koordination" und davon, daß beides einander abstimmen und ergänzen müsse, damit von einer integren Wissenschaft ausgegangen werden kann (siehe dazu auch Işcen 2002; in türkischer Sprache).
} 
105). Ohne auf die jeweilige Definition und Bestimmung dieser Begriffe und ihres Verhältnisses zueinander vorgreifend und dadurch die weitere Diskussion manipulierend einzugehen, sei das übersetzungswissenschaftlich spezifische Problem durch folgende Überlegung klar zum Ausdruck gebracht: Die Ausgewogenheit, die logische und natürliche, d.h. auch notwendige Abstimmung zwischen Koordination (Theoriebildung) und Subordination (Gegenstandsforschung) ist in der ÜW nicht gegeben. In bezug auf Subordination liegt kein Problem vor, nur wie diese subordinierenden Materialien koordiniert werden sollen, darin, in der begrifflichen Setzung dessen, wie Koordination bewerkstelligt werden kann, liegt das wissenschaftstheoretische Kernproblem. Ohne Koordination gibt es keine Wissenschaft (vgl. dazu Schopenhauer (1819/1977: 100-105). Alle (subordinativorientierten ${ }^{20}$ ) Erkenntnisse sind nur Anhäufung von Wissensmaterialien, die mit zunehmender Vielfalt (jede neue Untersuchung liefert ein neues Resultat, das oft mit dem vorhergehenden nicht referiert) nur den Bestand an Subordination aufballt, ins Unendliche hinausdehnt. Mit anderen Worten: erhöhte Subordination untergräbt jede Möglichkeit zur Koordination, die zunehmend erschwert wird, da es (d.i. die gegenstandsorientierte subordinierende Forschung) durchaus "ein weites Feld" (Snell-Hornby et al. 1999, Vorwort) ist.

Es gibt noch keine stichhaltige, allseits anerkannte Koordinanz innerhalb der ÜW. Es gibt nur eine zusehends unüberschaubar werdende, ungeordnete und regellose Anhäufung von Subordinaten, d.h., es "ist die einschlägige [übersetzungswissenschaftliche] Literatur inzwischen unüberschaubar - und täglich kommt Neues hinzu".

\section{Literaturangaben}

Ammann, Margret (1990): Grundlagen der modernen Translationstheorie - Ein Leitfaden für Studierende. Heidelberg.

Gerzymisch-Arbogast, Heidrun (1994): Übersetzungswissenschaftliches Propädeutikum. Tübingen/Basel.

Horn-Helf, Brigitte (1999): Technisches Übersetzen in Theorie und Praxis. Tübingen/Basel. Işcen, Ismail (2002): Çevrim Kuramı. Ankara: 65-80.

Kadric, Mira/Kaindl, Klaus/Pöchhacker, Franz (eds.) (2000): Translationswissenschaft. Festschrift für Mary Snell-Hornby. Tübingen.

Koller, Werner (1992): Einführung in die Übersetzungswissenschaft. Heidelberg/Wiesbaden. Lauer, Angelika/Gerzymisch-Arbogast, Heidrun/Haller, Johann/Steiner, Erich (eds.) (1996): Übersetzungswissenschaft im Umbruch. Festschrift für Wolfram Wills zum 70. Geburtstag. Tübingen.

Reiß, Katharina/Vermeer, Hans J. (1991): Grundlegung einer allgemeinen Translationstheorie. Tübingen.

Schäffner, Christiane (2000): "Kontinuität und Erneuerung". In: Schmitt, Peter A. (ed.): Paradigmenwechsel in der Translation. Festschrift für Albrecht Neubert zum 70. Geburtstag. Tübingen: 205-216.

\footnotetext{
${ }^{20}$ Siehe dazu als sehr aufschlussreiche Folgerungen in Stolze (2003: 30).
} 
Gedanken zum problematischen Selbstverständnis einer Übersetzungswissenschaft

Schopenhauer, Arthur (1977): Die Welt als Wille und Vorstellung I. Erster Teilband. Zürich.

Snell-Hornby, Mary/Hönig, Hans G./Kußmaul, Paul/Schmitt, Peter A. (eds.) (1999): Handbuch Translation. Tübingen.

Stolze, Radegundis (1994): Übersetzungstheorien. Tübingen.

Stolze, Radegundis (2003): Hermeneutik und Translation. Tübingen.

Vermeer, Hans J. (1983): Aufsätze zur Translationstheorie. Heidelberg 